\title{
A Continuous Representation of Heart Rate
}

\author{
K. Han, J.H. Nagel and N. Schneiderman \\ Behavioral Medicine Research Center, Department of Psychology and \\ Department of Biomedical Engineering, University of Miami, Coral Gables, FL 33124
}

\begin{abstract}
Analysis of heart rate variability (HRV) often requires a continuous representation of the inherently discrete heart rate measurement. In combination with a suitable cardiac pacemaker model, e.g. the integral pulse frequency modulator (IPFM), the cardiac event series can be considered as an irregular sampling of a continuous input to the pacemaker model, $m(t)$. Continuous representation of heart rate can thus be achieved by a reconstruction of the input function $m(t)$ from the cardiac event series. Two such representations of the heart rate, the instantaneous heart rate (IHR) and the low pass filtered event series (LPFES), have previously been assumed to be consistent with the IPFM model. Simulations show, however, that the LPFES actually is not consistent with the model. The IHR representation, although consistent with the model, suffers from discontinuities which are both unphysiological and inadequate for subsequent signal processing. A solution to the problem has been developed by introducing $M(t)$, the continuous integral of $m(t)$. The samples of $M(t)$ are specified by the cardiac event series and continuous representation of $\mathrm{M}(\mathrm{t})$ is achieved by cubic spline interpolation. The input to the cardiac pacemaker model $m(t)$, or in other words, the representation of the heart rate is given by the derivative of $M(t)$.
\end{abstract}

\section{Introduction}

Variations of heart rate, as a normal physiological phenomenon, reflect the activities of the cardiac control system. Various components of the heart rate variability (HRV) can be identified by spectral analysis. They are related to different cardiac control activities such as blood pressure, thermal regulation and respiration [5]. Sympathetic and parasympathetic origins have been identified for specific spectral components [1]. Thus, analysis of HRV can provide information on autonomic functions, especially the parasympathetic control of the heart [4]. However, because the measured heart rate is inherently a discrete signal, which is not defined between heart beats, study of HRV, especially by digital signal processing, often requires representation of the signal by a substitute continuous function of time in order to obtain regularly sampled data. Finding an adequate continuous function is possible only when enough knowledge and a model of the mechanism of the cardiac pacemaker is available.

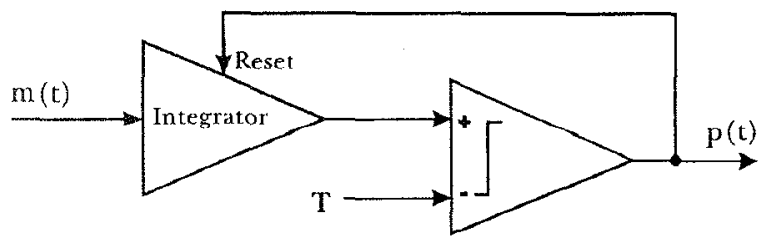

Figure 1: Diagram of the IPFM model.

A generally used model of the cardiac pacemaker is the integral pulse frequency modulator (IPFM) shown in Fig.1. The integration is performed on a positive input $m(t)$ representing the autonomic control activity to the heart and an impulse is initiated whenever the integral reaches a threshold $\mathrm{T}$. The integrator is then reset to zero and the integration restarts. This process generates an output impulse train $p(t)$ representing the cardiac event series [3].
For the cardiac event series $p(t)$, expressed as a sum of unit intensity impulses (Eq.1),

$$
p(t)=\sum_{k} \delta\left(t-t_{k}\right)
$$

the times of any two successive impulses, $t_{k}$ and $t_{k+1}$, are related to $m(t)$ by the integral equation:

$$
\int_{t_{k}}^{t_{k+1}} m(t) d t=T
$$

The significance of the IPFM model is that it relates the heart rate with a continuous signal representing the cardiac control activities and, therefore, provides a basis to derive a continuous representation of the heart rate. In other words, the discrete heart rate signal is represented by a continuous signal obtained through the reconstruction of $m(t)$ from the recorded cardiac event series. Two methods to obtain $m(t)$ from the cardiac event series are believed to be consistent with the IPFM model. With the first method, the instantaneous heart rate (IHR) is obtained by constant interpolation of the heart rate in the inter-beat intervals [2]. The IHR signal is a staircase signal with possible discontinuities at each heart beat. With the second method, a low pass filtered event series (LPFES) is computed by:

$$
f(t)=\sum_{k} \frac{\sin \left(2 \pi f_{c}\left(t-t_{k}\right)\right.}{\pi\left(t-t_{k}\right)}
$$

which is essentially an ideal low pass filtering of the impulse series with the cut-off frequency $f_{c}$. It has been claimed that the cardiac event series can be considered as the observation of $m(t)$ in irregular time intervals. Since the mean frequency of these observations is the actual mean heart rate, it follows that, according to the Nyquist criterion, the maximum observable frequency of $m(t)$ is limited to half the mean heart rate, though in reality, the maximum frequency is lower. With a mean heart rate of 60 beats per minute, i.e. $1 \mathrm{~Hz}$, the bandwidth of $\mathrm{m}(\mathrm{t})$ is always less than $0.5 \mathrm{~Hz}$. Therefore, the cut-off frequency $\mathrm{f}_{\mathrm{c}}$ may be chosen as $0.5 \mathrm{~Hz}$ [3]. Fig. 2 shows the IHR and LPFES representations of a heart rate signal. According to the IPFM model, a simple test can be performed by computer simulations. Provided that the integrator is reset at the time of a cardiac impulse, an output impulse train will be generated by the IPFM which will be the same as the original cardiac event series, if the calculated $m(t)$ is consistent with the model. As a testing result, Fig.3 shows the time differences between the impulse train received from $m(t)$ and the true cardiac event series. The IHR signal, though unphysiological and inadequate for digital signal processing, is consistent with the model since the generated impulse train is virtually the same as the cardiac event series. The maximum errors is $1 \mathrm{~ms}$, which corresponds to the time resolution of the computer simulations. It is obvious that the impulse train generated from the LPFES is significantly different from the original cardiac event series. Therefore, the LPFES signal actually is not consistent with the model.

\section{Cubic Spline Interpolation}

Since there is only one heart rate value corresponding to each interbeat interval regardless of the duration of the period, information concerning heart rate is inherently discrete. To obtain a continuous representation, some kind of interpolation between known samples of $\mathrm{m}(\mathrm{t})$ should be used. The first problem is to find the samples. According to Eq. 2 and the mean-value theorem, one can conclude that if $m(t)$ is 
continuous in the period $\left(t_{k}, t_{k+1}\right)$, there exists at least one $t_{\xi}$ for which

$$
m\left(t_{k}\right)\left(t_{k+1}-t_{k}\right)=T \quad t_{k} \leq t_{\xi} \leq t_{k+1}
$$

For the case of the IHR method, $\mathrm{m}(\mathrm{t})$ is assumed to be constant during each inter-beat interval, so there is no problem identifying $t_{\xi}$. In the more general case where $m(t)$ is not limited to a constant, $t_{\xi}$ is unknown. As a consequence, no sample points for $m(t)$ are readily available. Fortunately one can express $\mathrm{Eq} .2$ in term of $\mathrm{M}(\mathrm{t})$, the continuous integral of $\mathrm{m}(\mathrm{t})$. Let $\mathrm{t}_{0}=0$ be the time origin, then an equivalent expression for $\mathrm{Eq} .2$ is:

$$
M\left(t_{k}\right)=\int_{0}^{t_{k}} m(t) d t=k T
$$

As a function of time, the values of $M(t)$ at $t_{k}, k=1,2, \ldots, n$, are given by $k T$. From these given points, $M(t)$ can be reconstructed by interpolations and $\mathrm{m}(\mathrm{t})$ can be obtained by calculating the derivative of $M(t)$. If $M(t)$ is obtained by piece-wise linear interpolations, its derivative turns out to be the IHR signal. Although $M(t)$, in this case, is continuous at the joints, its derivative $\mathrm{m}(\mathrm{t})$ is not. To provide continuity to its higher order derivatives at the joints, cubic spline interpolation may be considered.

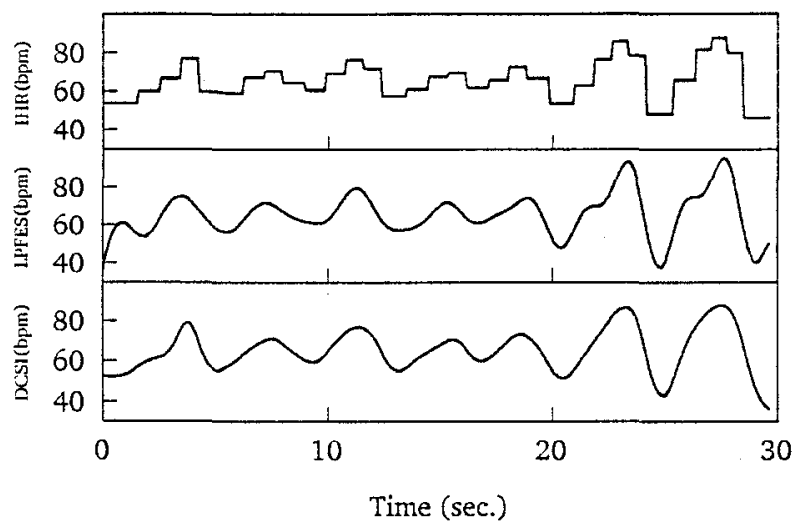

Figure 2: Representations of a heart rate signal

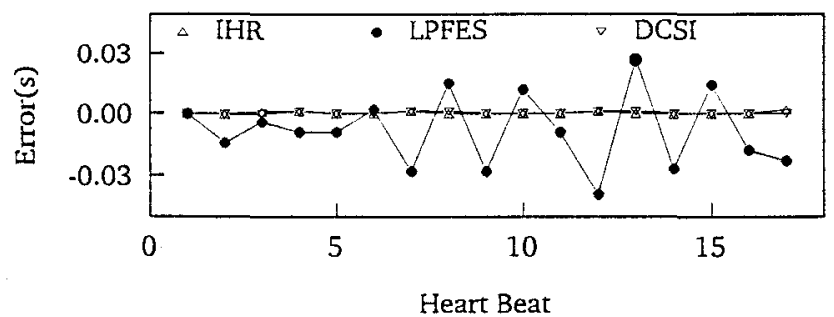

Figure 3: Errors in generated impulse trains

The spline interpolations are piece-wise polynomial interpolations in which low degree polynomials are chosen to pass each pair of given points and provide the maximum smoothness at joints. Cubic spline interpolation can provide continuity at joints up to the second order derivatives. As a widely used tool in engineering practice, the method of computing cubic spline interpolation has been well established. Because there is no information available outside the recording period, i.e. the first derivatives of $M(t)$ for the first and last points are not known, free-end cubic splines are used for the interpolation of $M(t)$. The two second derivatives at both ends required by the algorithm may be set to zero, provided that the trends of $\mathrm{m}(\mathrm{t})$ do not change at both ends of the period. After the spline functions are obtained, the derivation can be performed on these functions and the interpolations of $m(t)$, instead of $M(t)$, can be computed directly. The signal derived this way may be called the derivative of cubic spline interpolation or DCSI.
In summary, the procedure for the new method is: (1) Obtain the cardiac event series with a total of $n+1$ beats in the record occurring at $t_{0}, t_{1}, \ldots, t_{n}$ with $t_{0}=0$. (2) For the defined points $M\left(t_{k}\right)=k T, k=0,1$, ..., $\mathrm{n}$ and with the two second order derivatives at both end points equal to zero, calculate the coefficients of the $n$ free-end cubic splines $C_{i}(t)=$ $\alpha_{i}+\beta_{i}\left(t-t_{i}\right)+\gamma_{i}\left(t-t_{i}\right)^{2}+\delta_{i}\left(t-t_{i}\right)^{3}$, with $i=0,1, \ldots, n-1$, for the $\mathrm{n}$ intervals between consecutive points. (3) With the derivatives of the cubic splines, $m(t)$ is computed as:

$\mathrm{m}(\mathrm{t})=\Sigma \mathrm{C}_{\mathrm{i}}{ }^{\prime}(\mathrm{t})\left[\mathrm{u}\left(\mathrm{t}-\mathrm{t}_{\mathrm{i}+1}\right)-\mathrm{u}\left(\mathrm{t}-\mathrm{t}_{\mathrm{i}}\right)\right]$

with $C_{i}{ }^{\prime}(t)=\beta_{i}+2 \gamma_{i}\left(t-t_{i}\right)+3 \delta_{i}\left(t-t_{i}\right)^{2}$, for $t_{i} \leq t \leq t_{i+1}$ and $i=$ $0,1, \ldots, \mathbf{n}-1$.

\section{Results and Conclusion}

A DCSI representation of the heart rate is shown in Fig.2. The DCSI signals were tested by simulations and a result is shown in Fig.3. Similar to the IHR, the impulse trains from the DCSI signals are almost exactly the same as the recorded signals with a maximum error of $1 \mathrm{~ms}$, which is the resolution of computer simulation used. Except for this consistency with the IPFM model, the DCSII representation is continuous as shown in Fig.2. In conclusion, a continuous representation of the heart rate signal which is consistent with the IPFM model can be derived by cubic spline interpolations. Regularly sampled data representing HRV are readily obtained, and a variety of digital signal processing techniques may be applied in the analysis of the HRV.

Appendix: Computing Coelficients of Free-End Cubic Splines

Given $n+1$ points $\left\{t_{i}, x_{i}\right\}, i=0, \ldots, n$, and two second order derivatives at both end points: $2 q_{0}$ and $2 q_{n}$, the $n$ free-end cubic splines $C_{i}(t)=\alpha_{i}+\beta_{i}\left(t-t_{i}\right)+\gamma_{i}\left(t-t_{i}\right)^{2}+\delta_{i}\left(t-t_{i}\right)^{3}$

can be computed as following:

Let $\quad \Delta \mathrm{t}_{\mathrm{i}}=\mathrm{t}_{\mathrm{i}+1}-\mathrm{t}_{\mathrm{i}}$

$$
\Delta \mathrm{x}_{\mathrm{i}}=\mathrm{x}_{\mathrm{i}+1}-\mathrm{x}_{\mathrm{i}}
$$

and $\quad \Delta \mathrm{u}_{\mathrm{i}}=\Delta \mathrm{x}_{\mathrm{i}} / \Delta \mathrm{t}_{\mathrm{i}}$

Calculate $\mathrm{q}_{1}, \ldots, \mathrm{q}_{\mathrm{n}-1}$ by solving the $\mathrm{n}-1$ system of linear equations

$\left(\Delta \mathrm{t}_{0} \mathrm{q}_{0}+2\left(\Delta \mathrm{t}_{0}+\Delta \mathrm{t}_{1}\right) \mathrm{q}_{1}+\Delta \mathrm{t}_{1} \mathrm{q}_{2}=3\left(\Delta \mathrm{u}_{1}-\Delta \mathrm{u}_{0}\right)\right.$

$\Delta \mathrm{t}_{1} \mathrm{q}_{1}+2\left(\Delta \mathrm{t}_{1}+\Delta \mathrm{t}_{2}\right) \mathrm{q}_{2}+\Delta \mathrm{t}_{2} \mathrm{q}_{3}=3\left(\Delta \mathrm{u}_{2}-\Delta \mathrm{u}_{1}\right)$

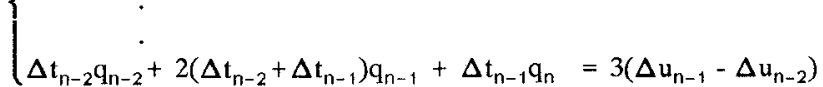

Note that $\mathrm{q}_{0}$ and $\mathrm{q}_{\mathrm{n}}$ are given parameters. Then the coefficients of the cubic splines for each interval can be obtained from the following formula:

$$
\begin{aligned}
& \alpha_{i}=x_{i} \\
& \beta_{i}=\Delta u_{i}-\Delta t_{i}\left(2 q_{i}+q_{i+1}\right) / 3 \\
& \boldsymbol{\gamma}_{i}=q_{i} \\
& \delta_{i}=\left(q_{i+1}-q_{i}\right) / 3 \Delta t_{i}
\end{aligned}
$$

\section{References}

[1] Akselrod, S., Gordon, D., Ubel, F.A. and et al., Power Spectrum Analysis of Heart Rate Fluctuation: A Quantitative Probe of Beatto-Beat Cardiovascular Control, Science, Vol.213, pp220-222, 1981.

[2] De Boer, R.W., Karemaker, J.M. and Strackee, J., Description of Heart-Rate Variability Data in Accordance With a Physiological Model for the Genesis of Heartbeats, Psychophysiology, Vol.22: pp147-155, 1985.

[3] Hyndman, B.W. and Mohn, R.K., A Puise Modulator Model of Pacemaker Activity, Digest of the 10th Int'l Conf. on Med. and Biol. Eng., Dresden, 1973, p223.

[4] Pomeranz, B., Macaulay, R.J.B., Caudill, A. and et al., Assessment of Autonomic Function in Humans by Heart Rate Spectral Analysis, American Journal of Physiology, Vol.248, (Heart Circ. Physiol. 17), ppH151-H153, 1985.

[5] Sayers, B.McA., Analysis of Heart Rate Variability, Ergonomics, Vol.16: pp17-32, 1973 .

Kedu Han, Department of Biomedical Engineering, University of Miami, P.O. Box 248294, Coral Gables, FL 33124, U.S.A. 